\title{
Optimum experimental conditions for quantitative surface microanalysis by reflection electron energy-loss spectroscopy
}

\author{
Zhong Lin Wang $\left({ }^{1,2}\right)$ and James Bentley $\left({ }^{1}\right)$ \\ ( $\left.{ }^{1}\right)$ Metals and Ceramics Division, Oak Ridge National Laboratory, P.O. Box 2008, Oak Ridge, TN \\ 37831-6376, U.S.A. \\ $\left({ }^{2}\right)$ Department of Materials Science and Engineering, The University of Tennessee, Knoxville, TN \\ 37996-2200, U.S.A.
}

(Received October 12, 1990; accepted March 26, 1991)

\begin{abstract}
Experimental conditions for obtaining high quality core-shell ionization edges in reflection electron energy-loss spectroscopy (REELS) are investigated. Under the (600) specular- "mirror" reflection conditions and using the relative ionization cross-section measured from a $\mathrm{MgO}$ thin foil in the transmission geometry for collection semi-angle $\beta=1.2 \mathrm{mrad}$, the chemical composition of $\mathrm{MgO}$ (100) surfaces is determined to be $N_{\mathrm{O}} / N_{\mathrm{Mg}}=1.5 \pm 0.15$. This value is not significantly affected by varying the resonance diffraction conditions near the [001] zone axis, under which the spectra were acquired. An incorrect apparent composition will result if channeling effects along the [011] zone axis are not considered properly. Surface microanalysis is limited by the accuracy of the core-shell effective ionization cross-section (EICS), which depends not only on the property of a single atom but also on the dynamical elastic and inelastic scattering and channeling processes of electrons. An experimental method is outlined by which to measure the relative EICS from a thin foil specimen in the transmission case under the equivalent resonance conditions as in reflection geometry.
\end{abstract}

\section{Introduction.}

Reflection electron energy-loss spectroscopy (REELS) combines the techniques of reflection electron microscopy (REM) and electron energy-loss spectroscopy (EELS) in a transmission electron microscope (TEM) [1]. The REELS spectra are acquired from reflected electrons under surface resonance conditions (see [2] for a review), the electrons having travelled a certain distance along the surface before being reflected [3]. Surface compositional microanalysis, an important application of REELS, usually requires the simultaneous detection of two or more atomic innershell ionization edges. As a result of strong dynamical scattering effects, however, the signal-tobackground $(S / B)$ ratios of the $\mathrm{K}$ ionization edges located above $1 \mathrm{keV}$ are limited in the REM geometry, which may compromise the accuracy of surface microanalysis.

In REM, the electrons reflected from the surface can be classified as Bragg-but not resonance, resonance-but not Bragg, and Bragg-resonance. The optimum inelastic signal usually obtained in the last case. In practice, there are many ways to achieve surface resonance, such as axial and 
planar channeling at different incidence angles. The incidence angle has to be properly chosen in order to avoid strong multiple valence excitations at low angles and deep penetration into the surface at high angles. The strongest reflected intensity may not always be accompanied by the optimum inelastic signal, because the accuracy of surface microanalysis is affected by the $S / B$ ratio, which depends strongly on the diffracting conditions.

In this paper, an optimum diffracting condition is described, based on comparisons of spectra acquired from a $\mathrm{MgO}$ (100) surface under different resonance conditions. The effects of several experimental parameters on the accuracy of surface compositional analysis are investigated. The complexity of REELS microanalysis and possible experimental solutions are discussed. The effects of surface channeling are also illustrated.

\section{Experimental conditions.}

The REM and REELS experiments were performed in a Philips CM30 (300 kV) TEM. The REELS spectra were collected by a parallel detection system (Gatan 666 PEELS spectrometer) at an energy resolution of about $3 \mathrm{eV}$. Core ionization edge spectra were typically acquired in about 40-80 s. Bulk single crystal $\mathrm{MgO}$ specimens, about $2.5 \mathrm{~mm} \times 1.5 \mathrm{~mm} \times 0.7 \mathrm{~mm}$, were freshly cleaved in air along [001] just before examination by electron microscopy.

In early REELS studies of MgO (100) surfaces by Wang and Cowley [1] using a Gatan 607 serial detection system, the major obstacle was to obtain a reasonable signal-to-noise ratio, because it took about 10 minutes to acquire a core loss spectrum. However, in the present study with a parallel detection system, the major emphasis is to find diffracting conditions under which optimum $S / B$ ratios can be obtained.

\section{Optimum diffracting conditions.}

The $S / B$ ratio in REELS is determined by the diffracting conditions, which should provide inelastic signals with high surface sensitivity and REM images with reasonable contrast. Figure 1 shows several cases of reflection high-energy electron diffraction (RHEED) patterns taken from a $\mathrm{MgO}$ (100) surface under different resonance conditions with a beam azimuth close to [001]. Figures 2 and 3 show corresponding REM images and valence excitation spectra, respectively, for each case shown in figure 1 . For comparison purposes, figure $3 \mathrm{e}$ shows a spectrum acquired from a $\mathrm{MgO}$ thin foil using transmitted electrons, which represents the bulk rather than surface excitation of $\mathrm{MgO}$. The position of the $20 \mathrm{eV} \mathrm{MgO}$ "volume plasmon" is indicated with a vertical bar in each spectrum. Discussion of each diffraction condition can be classified as follows.

Case a: Figure 1a was a common resonance condition used for REELS analysis [1]. The (400) specularly reflected spot coincides with the (400) and (020) Kikuchi (K) lines; the (420) spot is simultaneously excited. Since strong dynamical effects are involved in this case, and the (400) spot is divided into several intensity sectors by the $\mathrm{K}$ lines [4], the image contrast is relatively poor (Fig. 2a). Also the low incidence angle of the electrons produces strong valence excitations (Fig. 3a), which increase the background level in the energy-loss range of core shells. In general, valence excitations in REELS have two contributions. One part is the delocalized excitation of the surface when the electrons approach and leave the surface. The probability of this process is inversely proportional to the grazing incidence angle [5]. The other part is the excitation of the surface and the bulk when the electrons are trapped in the crystal during the resonance process. The localized core shell excitations occur only when the electrons are propagating inside the crys-

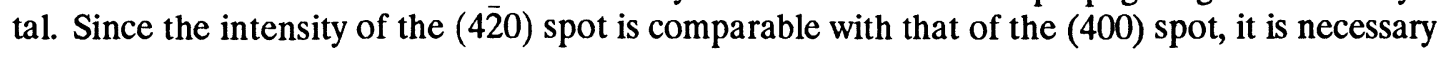



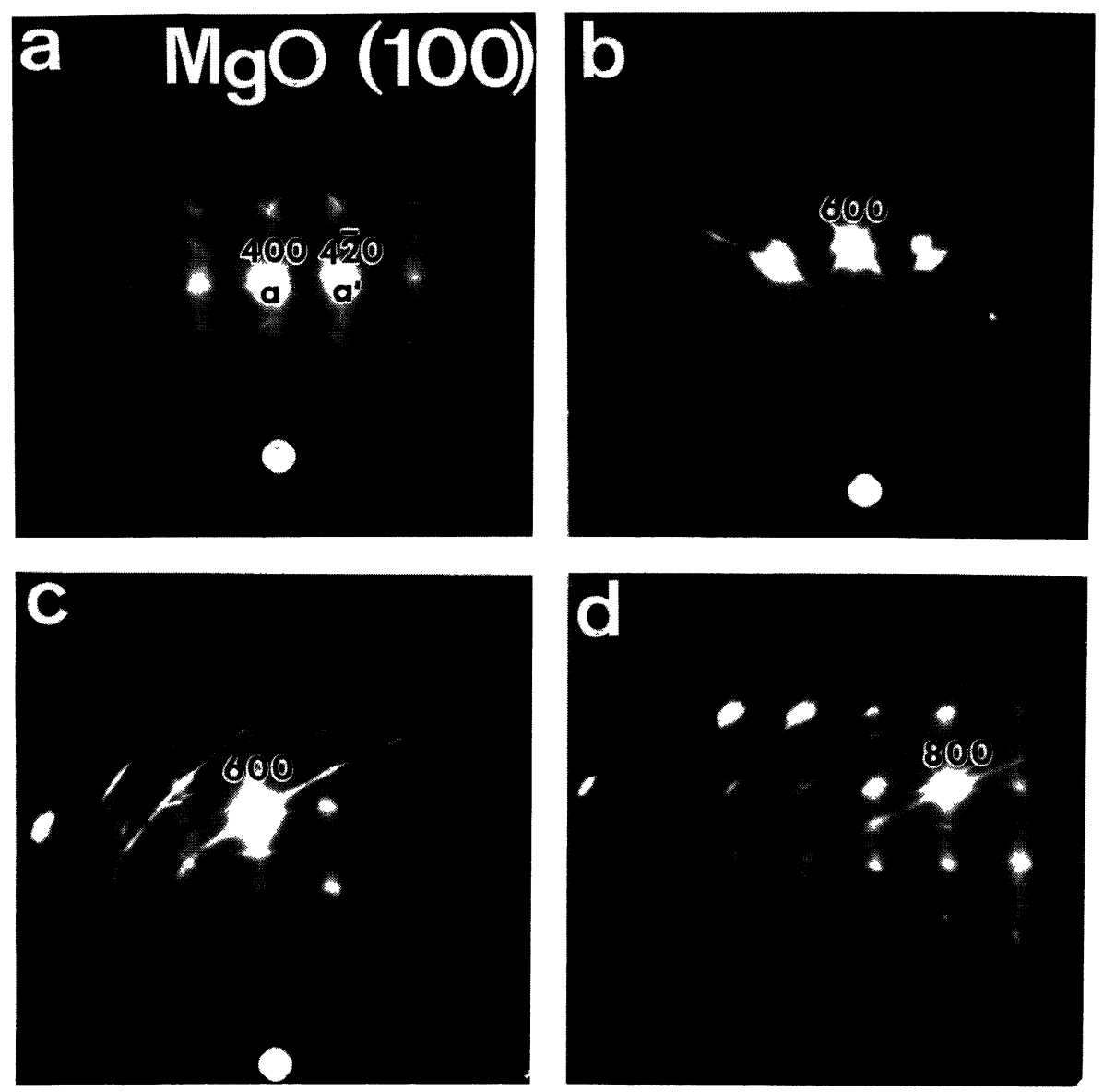

Fig. 1. - RHEED patterns from a $\mathrm{MgO}(100)$ surface under different resonance reflection conditions. Beam azimuth $B \approx[001]$.

to consider the contribution of the tail of the (4⿳亠丷厂二 0$)$ spot in the EELS collection aperture. Such effects can introduce a 5-10\% correction to the apparent $\mathrm{MgO}$ surface composition $[2,6,7]$.

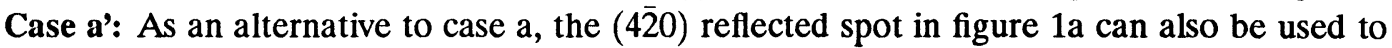
acquire REELS spectra. As well as poor REM image contrast (Fig. 2a'), the valence excitations are also increased (Fig. 3a').

Case b: To decrease multiple valence excitations (see Fig. 3b), the electron incidence angle can be increased so that the (600) spot is specularly reflected (Fig. 1b). With the electron azimuth parallel to the zone axis, the REM image contrast is optimized and the image "distortion" due to off axis incidence is minimized (Fig. $2 b_{1}$ and $b_{2}$ ). Since the MgO (100) surface was formed by cleavage along [001], the surface steps so formed are parallel to the [001] direction. As well as the (600) spot, the (620) and (620) spots are also strongly excited in figure 1b. Symmetrical diffracting conditions may complicate surface microanalysis because it is possible that row channeling effects 

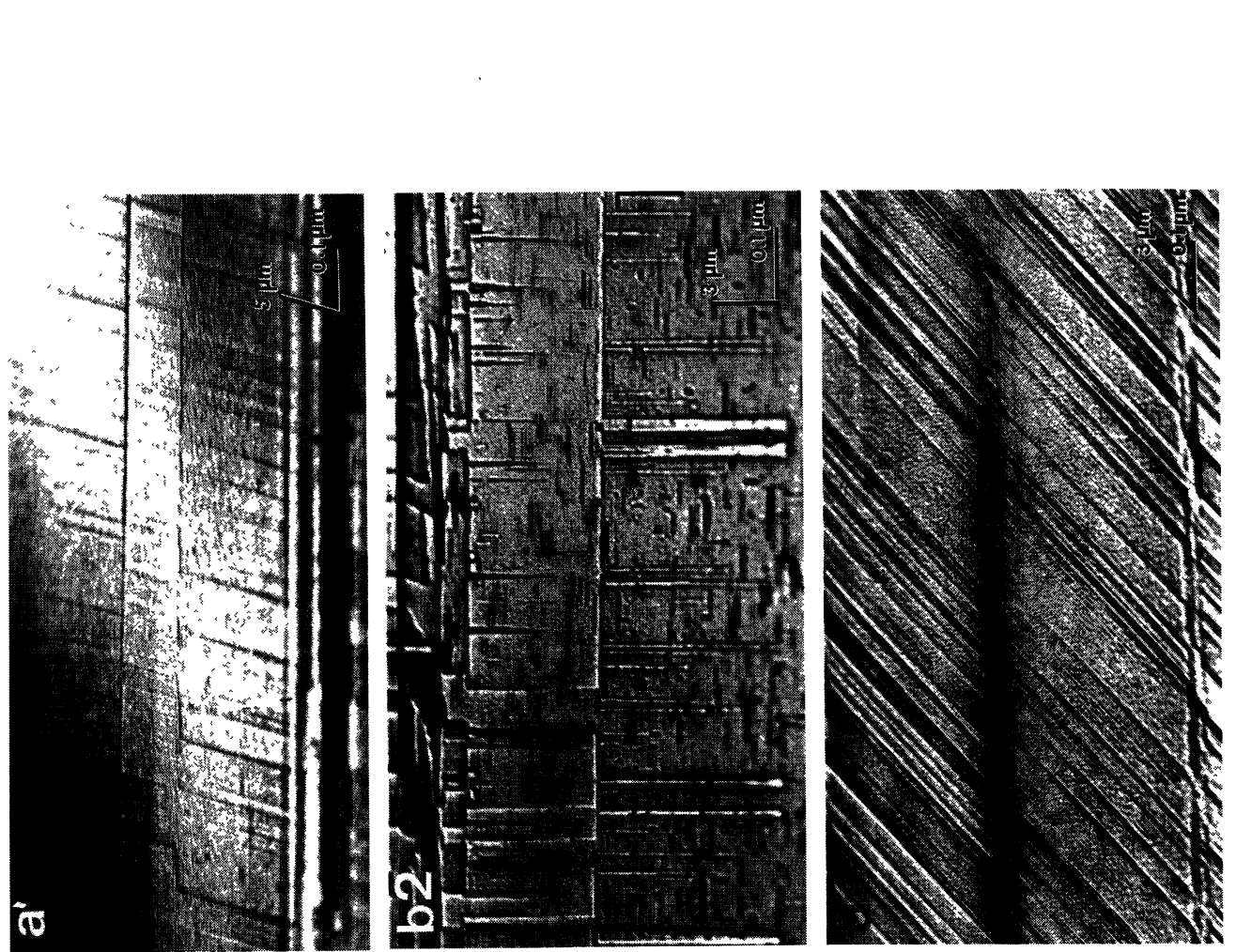

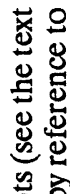

है

马

응

这

s.

5

害

7

늘

틍

. 8

है

동 8

n

올

藏

ठ․

용

늘

象电
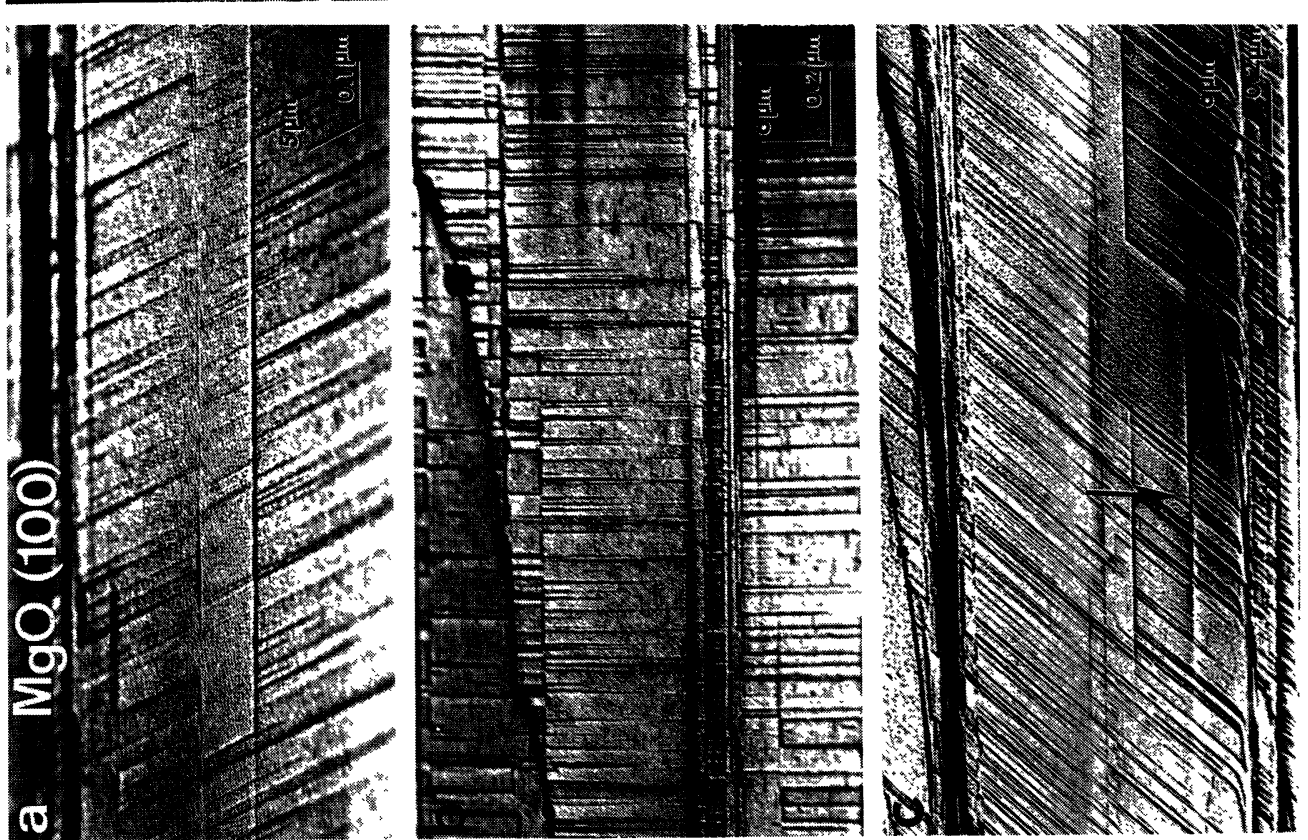

올

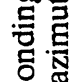

家

它

요

讨

당

등

웡

현

ษ 인

灵 믐

ริํํ응

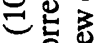

잉

हते

䒕

응물

过

跑氙

昰焉

1 흘

츄

워

क் 

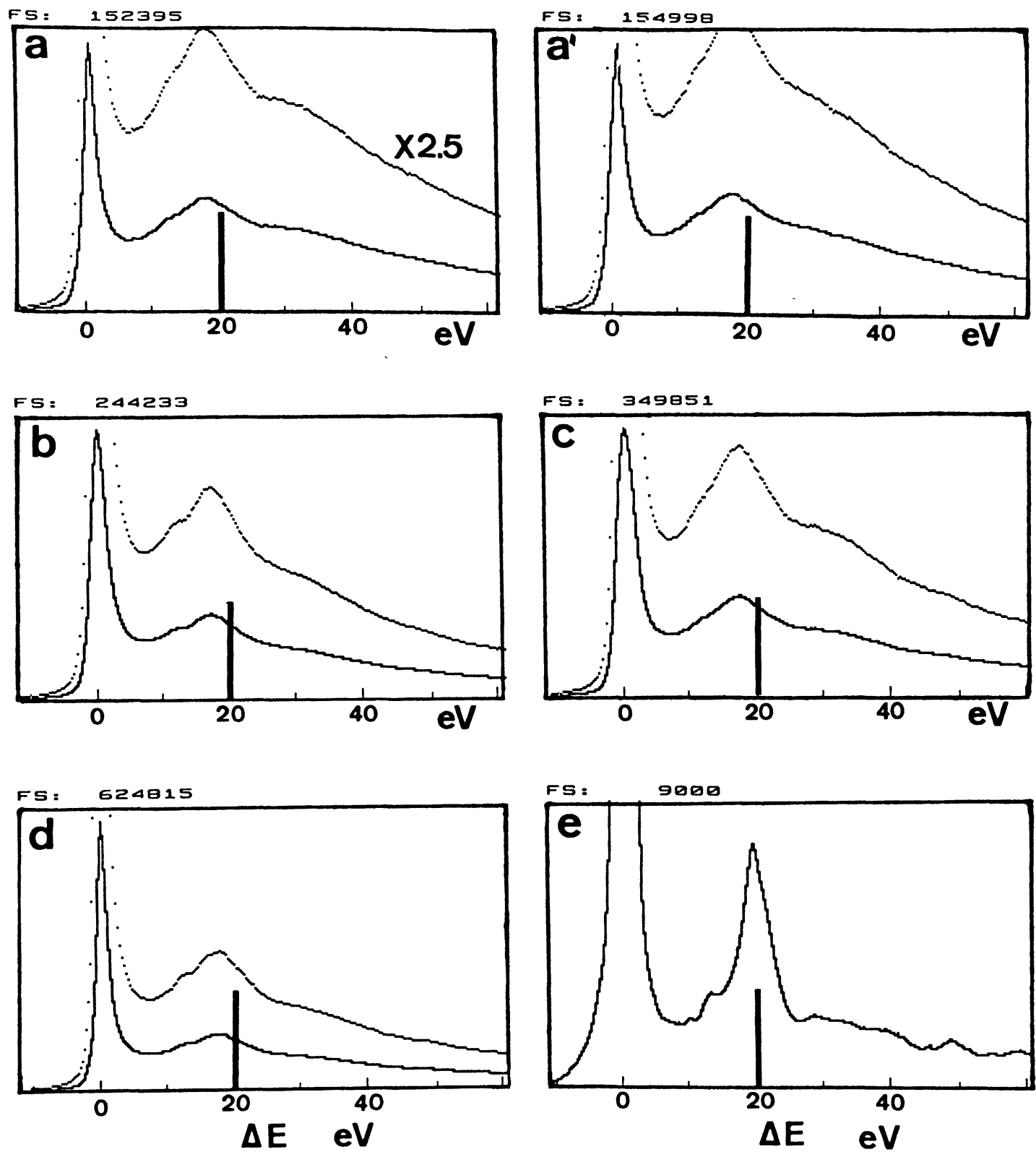

Fig. 3. - Low energy-loss valence excitation spectra acquired from a $\mathrm{MgO}$ (100) surface under the corresponding diffraction conditions shown in figure 1 for $\beta=1.2 \mathrm{mrad}$ (see the text for details). The spectrum displayed in e) was acquired from a thin $\mathrm{MgO}$ foil in the transmission geometry and characterizes the bulk excitation. Dotted spectra are magnified 2.5 times, in each case.

will be important in this situation.

Case c: The strong diffracting condition in case $b$ can be decreased by tilting the incident beam off the zone axis in the direction parallel to the surface, so that only the specularly reflected spot is strongly excited (Fig. 1c). In this case, the (600) spot can be approximately considered as the 
"mirror" reflection. The corresponding REM image is "distorted", but the surface steps can be identified as being of atomic-height by reference to a screw dislocation arrowed in figure $2 \mathrm{c}$. Valence excitation is reasonably low in this case (Fig. 3c). It is again possible to have planar channeling effects in this case, but this should not affect the microanalysis of $\mathrm{MgO}(100)$ when beam azimuth $B \approx[001]$.

Case d: The valence excitation can be further decreased (see Fig. 3d) by increasing the electron incidence angle so that the (800) spot is strongly excited (Fig. 1d). This increases the depth to which the electrons penetrate the crystal and thus decreases the surface sensitivity of REELS, but still allows reasonably good REM images to be obtained (Fig. 2d).

As a summary of figures 1 to 3 , it seems that the best condition for REM imaging is case $b$ (Fig. $1 b$ ) and that cases $c$ and $d$ may be reasonable choices for acquiring REELS spectra.

Figure 4 shows the integrated $S / B$ ratios of the O-K and $\mathrm{Mg}-\mathrm{K}$ edges acquired under the various diffracting conditions of figure 1 for different EELS collection semi-angles. For small aperture sizes $(0.4 \mathrm{mrad})$, case $\mathrm{d}$ may be a reasonable choice for collecting the O-K edge but not for the $\mathrm{Mg}-\mathrm{K}$ edge. This is probably because the characteristic inelastic angle $\left(\theta_{\mathrm{E}}=\Delta E / 2 E_{0}\right.$, where $\Delta E$ is the energy-loss and $E_{0}$ is the incident electron energy) for $\mathrm{Mg}-\mathrm{K}$ is larger than that for $\mathrm{O}-\mathrm{K}$. The optimum $S / B$ ratio seems to be obtained for O-K and $\mathrm{Mg}-\mathrm{K}$ edges at diffraction case c when $\beta=$ $1.2 \mathrm{mrad}$. It is found experimentally that the $\mathrm{Mg}-\mathrm{K} S / B$ ratio becomes worse if $\beta>2 \mathrm{mrad}$. This is possibly because the anisotropic intensity distributions of the resonance parabola and Kikuchi lines become important at large collection angles, and consequently contribute a strong background at high energy losses (see Sect. 7 for details). This may indicate that the angular distribution of the core-shell inelastically scattered electrons is not proportional to the distribution of electrons that have suffered plasmon losses.
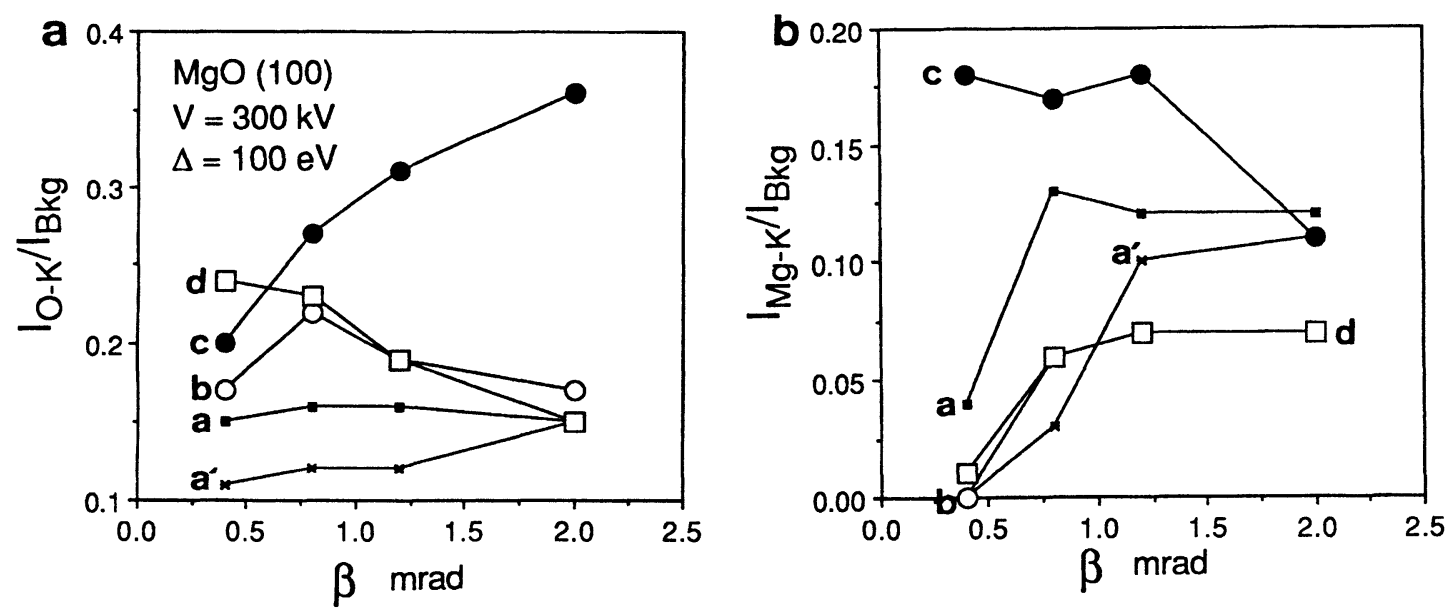

Fig. 4. - Dependences of signal-to-background ( $S / B$ ) ratios for (a) O-K and (b) Mg-K edges on diffraction conditions and spectrometer collection semi-angles. The measurements corresponding to the diffracting conditions shown in figure 1 are labeled with a, a', b, c and d, respectively. In (b), the curves for cases b and $\mathrm{d}$ are overlapped after $\beta>0.8 \mathrm{mrad}$. 


\section{Surface compositional microanalysis.}

Figure 5 shows REELS spectra of O-K and Mg-K edges acquired from a MgO (100) surface under diffracting condition $\mathrm{c}$ before and after the subtraction of background, with $\beta=1.2 \mathrm{mrad}$. Some near-edge structure is evident, but features in the extended energy-loss region are smeared out by multiple valence excitations. However, to a first order approximation, surface compositional analysis is not affected.
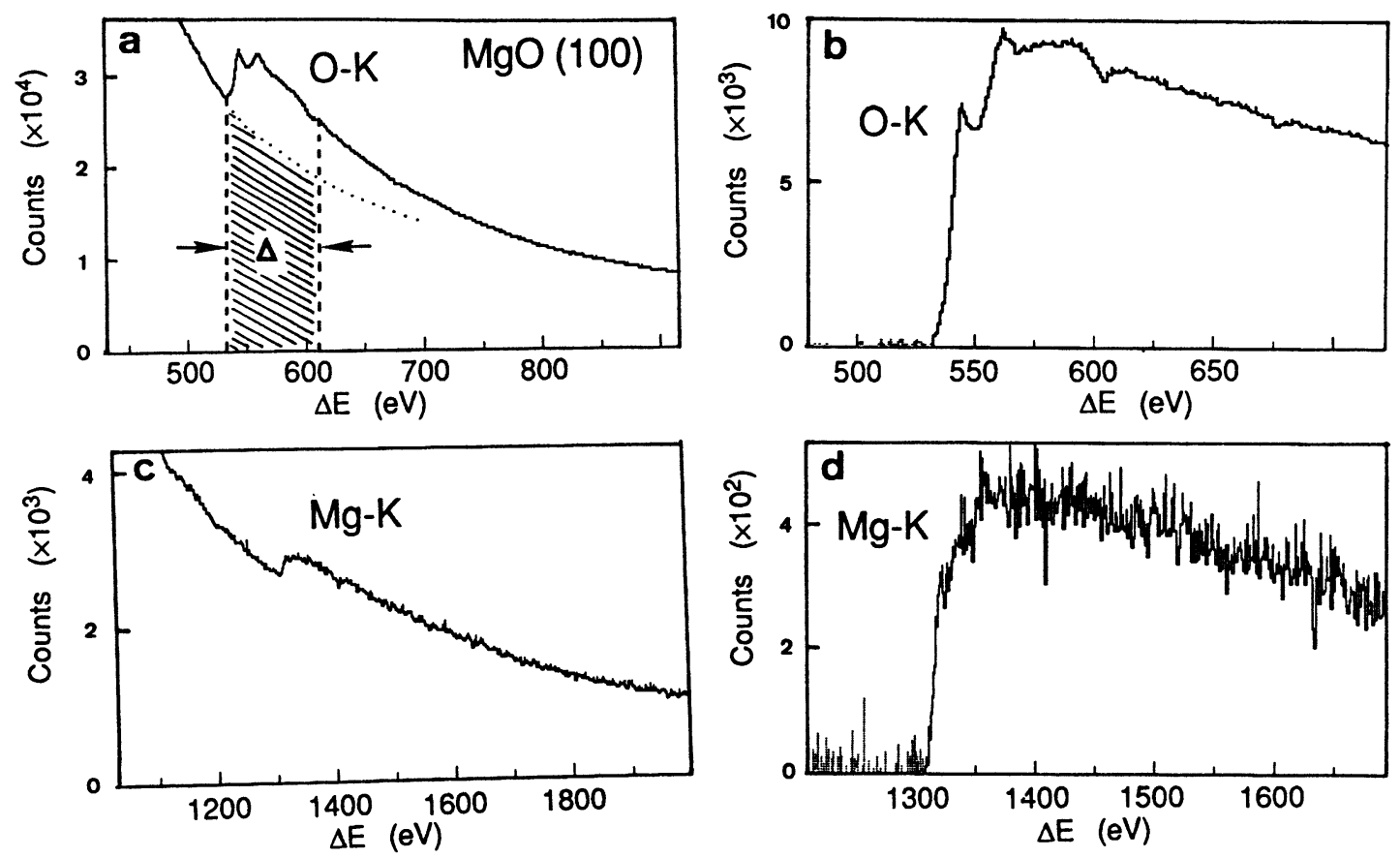

Fig. 5. - REELS spectra of O-K and Mg-K edges acquired under diffraction case c (Fig. 1c) from a MgO (100) surface ( $a$ and c) before and (b and d) after the subtraction of background. The (600) specular reflection spot was selected and $\beta=1.2 \mathrm{mrad}$. Beam azimuth $B \approx[001]$.

Under strong diffracting conditions, the intensity of an atomic core-shell edge acquired in diffraction mode can be written as,

$$
I_{\mathrm{A}}(\beta, \Delta)=\int_{0}^{D} \mathrm{~d} z \int_{\Sigma} \mathrm{d} x \mathrm{~d} y i(\mathbf{r}) n_{\mathrm{A}}(\mathbf{r}) \sigma(\mathrm{A}, \beta, \Delta)
$$

where $\sigma(\mathrm{A}, \beta, \Delta)$ is the single-atom ionization cross-section of element $\mathrm{A}$ for collection semiangle $\beta$ and energy window $\Delta ; n_{\mathrm{A}}(\mathbf{r})$ is the local atomic concentration of $\mathrm{A}$ atoms; $i(\mathbf{r})$ is the local channeling current density; $D$ is the mean distance electrons travel along the surface; and $\Sigma$ indicates a surface integration over the beam illumination area $S$. In the approximation of perfectly localized excitation, one has

$$
n_{\mathrm{A}}(\mathbf{r})=\sum_{j \mathrm{~A}} \delta\left(\mathbf{r}-\mathbf{R}_{j \mathrm{~A}}\right)
$$


where $\mathbf{R}_{j \mathrm{~A}}$ is the position of the $j^{\text {th }} \mathrm{A}$ atom in the crystal. By defining an effective ionization cross-section (EICS) as

$$
\sigma_{\mathrm{eff}}(\mathrm{A}, \beta, \Delta) \equiv \sigma(\mathrm{A}, \beta, \Delta) \frac{\sum_{j \mathrm{~A}} i\left(\mathbf{R}_{j \mathrm{~A}}\right)}{i_{0} N_{\mathrm{A}}} K_{\mathrm{A}} \equiv \sigma(\mathrm{A}, \beta, \Delta) \frac{\overline{i_{\mathrm{A}}}}{\overline{i_{0}}} K_{\mathrm{A}}
$$

where $i_{0}$ is the average current density; $N_{\mathrm{A}}$ is the average atomic concentration of atom $\mathrm{A} ; \overline{i_{\mathrm{A}}}$ is the average channeling current density at the $\mathrm{A}$ atomic sites; and $K_{\mathrm{A}}$ is introduced to take into account the deviation of the final inelastic electron angular distribution, $f(\alpha)$, from the Lorentzian function, $L(\alpha)$, due to dynamical diffraction effects. By assuming that the generalized oscillator strength is almost independent of the scattering angle $\alpha$,

$$
K_{\mathrm{A}}=\int \mathrm{d} \alpha f\left(\alpha, \theta_{\mathrm{E}}(\mathrm{A})\right) / \int \mathrm{d} \alpha L\left(\alpha, \theta_{\mathrm{E}}(\mathrm{A})\right),
$$

where the integration of $\alpha$ is limited to the collection aperture of semi-angle $\beta, \theta_{\mathrm{E}}(\mathrm{A})$ is the characteristic inelastic angle of the element $\mathrm{A}$ and $L$ is the angular distribution of the electrons after being inelastically scattered by a single atom,

$$
L\left(\alpha, \theta_{\mathrm{E}}\right)=1 /\left[\alpha^{2}+\theta_{\mathrm{E}}^{2}\right] .
$$

The compositional ratio for two elements is thus

$$
\frac{N_{\mathrm{A}}}{N_{\mathrm{B}}}=\frac{I_{\mathrm{A}}(\beta, \Delta)}{I_{\mathrm{B}}(\beta, \Delta)} \frac{\sigma_{\mathrm{eff}}(\mathrm{B}, \beta, \Delta)}{\sigma_{\mathrm{eff}}(\mathrm{A}, \beta, \Delta)}=\frac{I_{\mathrm{A}}(\beta, \Delta)}{I_{\mathrm{B}}(\beta, \Delta)} \frac{\sigma(\mathrm{B}, \beta, \Delta)}{\sigma(\mathrm{A}, \beta, \Delta)} \frac{\overline{i_{\mathrm{B}}} K_{\mathrm{B}}}{\overline{i_{\mathrm{A}}} K_{\mathrm{A}}} .
$$

It must be pointed out that the newly defined EICS is determined not only by the property of each single atom $(\sigma)$ but also by both the dynamical elastic and inelastic electron scattering $\left(K_{\mathrm{A}}\right)$ and the detailed channeling processes $\left(\overline{i_{\mathrm{A}}}\right)$ of the electrons. In REELS, for a general case, $\overline{i_{\mathrm{A}}} \neq$ $\overline{i_{\mathrm{B}}}$ and $K_{\mathrm{A}} \neq K_{\mathrm{B}}$, because the elastic Bragg reflections and core-shell ionizations may not be independent events. This complicates the analysis of REELS data (see Sect. 7 for details). In other words, calculated ionization cross-sections based on isolated atom properties are insufficient for REELS microanalysis; dynamical diffraction effects $\left(K_{\mathrm{A}}\right)$ and channeling discrimination effects $\left(\overline{i_{\mathrm{A}}}\right)$ generally have to be included. At this time it is not practical to calculate the latter two effects quantitatively, but it may be possible to measure their combined effects experimentally (see Sect. 7.2).

Equation (6) was derived by assuming that the intensities of other diffracted spots are much smaller than that of the specularly reflected spot. If this is not the case, a small correction has to be made in equation (6) $[6,7]$.

For MgO (100), since the oxygen and magnesium atoms are arranged alternately in the same row when the beam azimuth is close to [001], surface channeling (or resonance) effects, if any, should not affect the results of surface microanalysis (i.e., one can take $\overline{i_{\mathrm{A}}}=\overline{i_{\mathrm{B}}}$ in equation (6)). For $E_{0}=300 \mathrm{kV}, \Delta=100 \mathrm{eV}$ and $\beta=1.2 \mathrm{mrad}$, the ratio of ionization cross-sections measured using the (000) reflection spot in the transmission case of a thin $\mathrm{MgO}$ foil is $\sigma(\mathrm{O}, \beta, \Delta) / \sigma(\mathrm{Mg}, \beta, \Delta)=$ $(15.0 \pm 1.0)$. This value is used for following analysis when $\beta=1.2 \mathrm{mrad}$. 
Before one presents the experimental results, it is necessary to discuss the meaning of surface chemical compositions if there are some adsorbates. If one assumes there is a monolayer of oxygen being adsorbed on the $\mathrm{MgO}(100)$ surface, the ratio of $N_{\mathrm{O}} / N_{\mathrm{Mg}}$ would depend on the conditions under which the measurements were made, because the ratio of the inelastic signals provided by the incident electrons rely on the electron penetration depth into the surface. If the electrons penetrate up to only the first mixing O-Mg (100) layer, for an example, the measured surface composition would be $N_{\mathrm{O}} / N_{\mathrm{Mg}}=2$. If the electrons penetrate into the surface up to two mixing O-Mg layers, the surface composition determined would be $N_{\mathrm{O}} / N_{\mathrm{Mg}}=3 / 2$. Therefore, the surface composition may be a function of the electron penetration depth if there are some surface adsorbates. This is different from the composition measured in transmission EELS, being an absolute value. It is very important to remember this point when using the REELS measured results.

\section{Dependence of measured surface compositions on experimental parameters.}

5.1 DifFRACTING CONDITIONS. - As shown in figure 4, the $S / B$ ratios of core edges depend strongly on the diffraction conditions and cannot be predicted by a simple model. But it is surprising to find that the measured $\mathrm{MgO}(100)$ surface composition varies only slightly for the diffraction conditions shown in figure 1 , probably because channeling effects do not discriminate between $\mathrm{O}$ and $\mathrm{Mg}$ atoms when the beam azimuth is close to [001]. The result for $\beta=1.2 \mathrm{mrad}$ is $N_{0} / N_{\mathrm{Mg}}=$ $(1.5 \pm 0.15)$. A $50 \%$ increase of oxygen at the surface is indicated. This is in agreement with previous studies $[1,8,9]$.

5.2 EELS SPECTROMETER COLLECTION SEMI-ANGLE. - Table I shows the MgO (100) surface composition determined for diffraction case $c$ at different collection semi-angles. The relative ionization cross-sections for different $\beta$ angles were measured from a thin $\mathrm{MgO}$ foil in the transmission geometry. For small apertures $(\beta=0.4 \mathrm{mrad})$, the poor signal-to-noise ratio of the $\mathrm{Mg}$-Kedge limits the accuracy of the microanalysis. At large collection angles $(\beta=2.0 \mathrm{mrad})$, the anisotropic distribution of the inelastically scattered electrons in the reflected spot deviates strongly from the angular distribution in the transmission case, for which the ionization cross-section ratio was determined. It may be possible that the electrons scattered to different angular range come from different surface depth, resulting in the shift of the surface composition as observed in table I.

Table I. - Average surface compositions of MgO (100) determined under different EELS spectrometer collection semi-angles in diffraction condition c (Fig. 1c).

\begin{tabular}{|c|c|c|c|c|}
\hline$\beta(\mathrm{mrad})$ & 0.4 & 0.8 & 1.2 & 2.0 \\
\hline$N_{\mathrm{O}} / N_{\mathrm{Mg}}$ & $0.95 \pm 0.2$ & $1.3 \pm 0.15$ & $1.5 \pm 0.15$ & $2.0 \pm 0.1$ \\
\hline
\end{tabular}

Considering the large noise effect at small $\beta$ angles $(0.4 \mathrm{mrad})$ and the anisotropic angular distribution of the inelastically scattered electrons at large $\beta$ angles $(2.0 \mathrm{mrad})$, the composition measured at $\beta=1.2 \mathrm{mrad}$ may be a reliable in reference to our previouse studies $[1,10]$.

5.3 SURFACE STRUCTURES. - The intensity and shape of the O-K edge was found to depend on the structure of $\mathrm{MgO}(100)$ surfaces. For atomically flat surface areas, the intensity of the core 
edges were usually stronger than those acquired from areas with many steps, as shown in figure 6, possibly because the mean distance electrons travel is longer for a perfect surface than for a surface with many steps. The apparent surface composition $N_{\mathrm{O}} / N_{\mathrm{Mg}}$ varied from 1.4 to 1.5 for areas with and without many surface steps. For some rough surface areas, the apparent composition was as low as 1.1. This can be explained by the definition of surface composition discussed above.
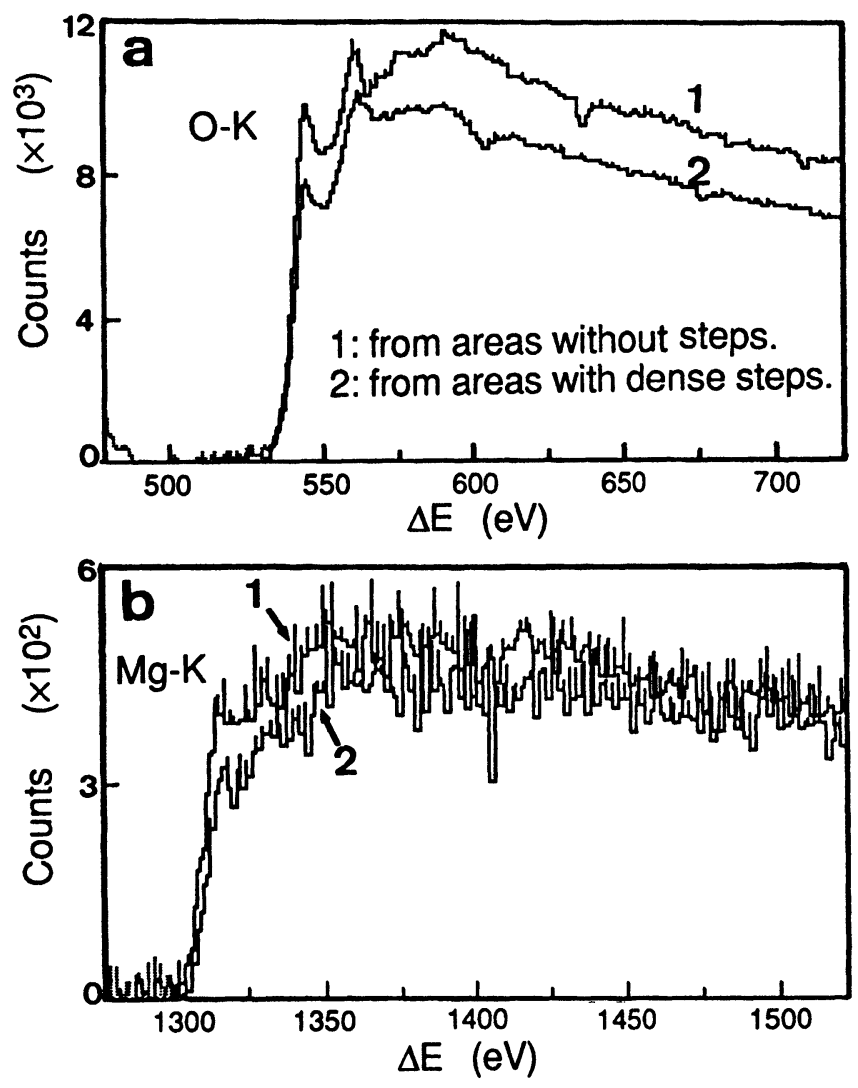

Fig. 6. - Sensitivity of REELS spectra to surface structures. Comparisons of (a) the O-K and (b) Mg-K edges acquired from surface areas with and without many surface steps. The acquisition time was $40 \mathrm{~s}$, diffraction case $\mathrm{c}$ (Fig. 1c) was selected, $\beta=1.2 \mathrm{mrad}$ and beam azimuth $B \approx[001]$.

5.4 OTHER PARAMETERS. - Surface compositions determined from REELS can also be affected by other parameters. The beam convergence can give a 5\% uncertainty. In practice, it is found that the $S / B$ ratio can be improved by the use of a convergent beam. Spectra acquired with and without the selected area aperture can also give a $5 \%$ difference. This may be due to the chromatic aberration effects for different energy losses [11].

\section{Channeling effects.}

As shown in figure 1, strong diffracting conditions are always involved in REELS microanalysis. Thus surface channeling (or resonance) effects are inevitably involved in enhancing the inelastic 
signal. It is possible to incorrectly determine surface compositions if the channeling effects produce differences in the excitations of different atoms arranged in separated rows or planes. This can happen for a $\mathrm{MgO}(100)$ surface if the incident beam azimuth is approximately parallel to [011]. Figure 7 shows the O-K and $\mathrm{Mg}-\mathrm{K}$ edges acquired from the $\mathrm{MgO}(100)$ surface under the diffracting conditions shown in the insert. The intensity ratio of $\mathrm{O}-\mathrm{K}$ to $\mathrm{Mg}-\mathrm{K}$ decreases by about a factor of 2 compared to those shown in figure 5. For $B=[011]$, an apparent surface composition of $N_{\mathrm{O}} / N_{\mathrm{Mg}}=0.7$ to 1.0 was obtained, if the relative scattering cross-section measured for $B=$ [001] is used. The higher Mg-K signal (relative to the $\mathrm{O}-\mathrm{K}$ ) is produced by strong channeling of the incident beam along the $\mathrm{Mg}$ atomic rows. It is obvious that the composition determined in this case is incorrect, because the discrimination between the $\mathrm{O}$ and $\mathrm{Mg}$ atoms by the electron channeling effects has not been taken into account. In some cases, however, channeling effects can be used to measure the amount of the surface adsorbates [12].

ORNL PHOTO 6311-90
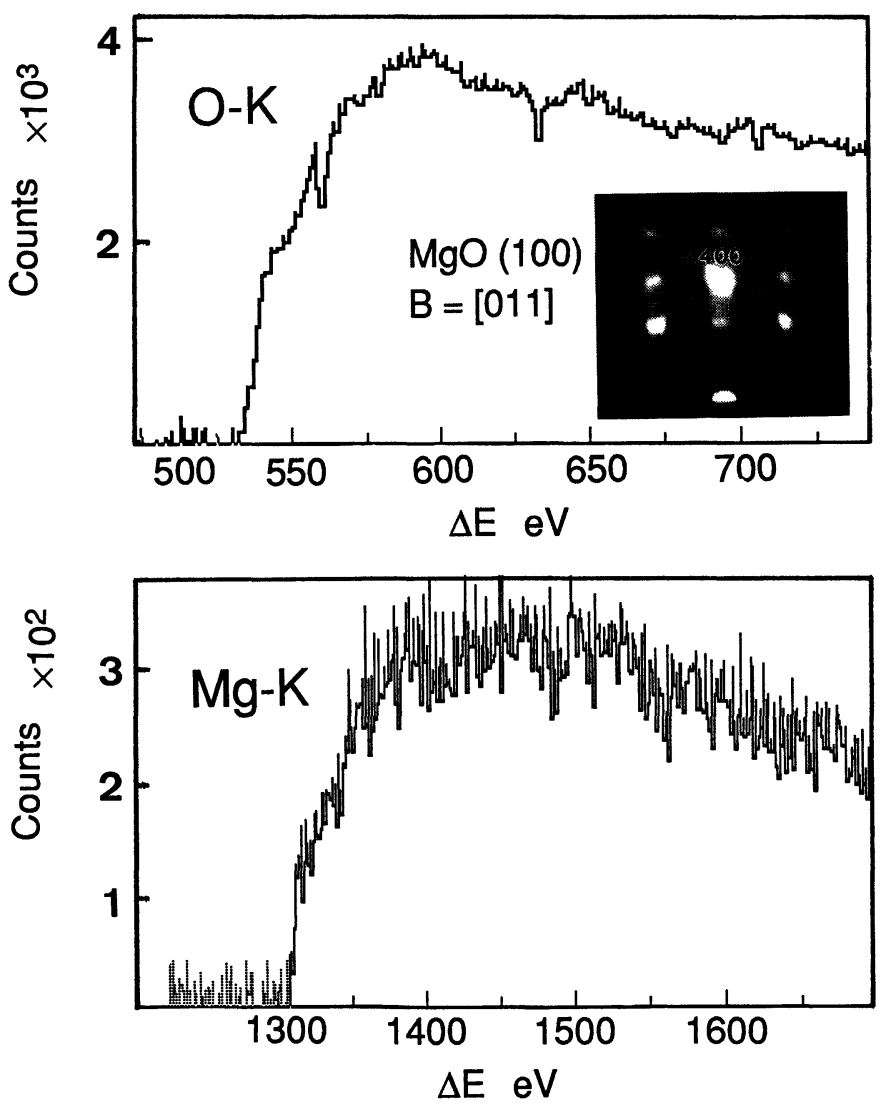

Fig. 7. - Channeling effects in REELS microanalysis. Background subtracted (a) O-K and (b) Mg-K edges acquired from the same $\mathrm{MgO}$ (100) specimen used for obtaining figure 5 except the surface was rotated so that the beam azimuth $B \approx[011]$. The insert is the corresponding RHEED pattern. 


\section{Discussion.}

7.1 DEPENDENCE of EICS on ELECTRON CHANNELING PROCESS. - As pointed out above, REELS surface microanalysis is mainly limited by the accuracy of the effective ionization crosssections that are related to the angular distribution function, $f(\theta)$, of the inelastically scattered electrons inside the reflected spot and the dynamical scattering process of electrons from the surface. The $f(\theta)$ function is the result of dynamical multiple elastic and inelastic electron scattering under the specified diffraction conditions and cannot be described by a Lorentzian function as in the transmission case under the kinematic approximation. To illustrate this point, one assumes that the electrons are channeling within the top two atomic layers under the Bragg-resonance reflection case, as shown in figure 8 [13]; the transmitted and reflected coefficients of the wave through and from each layer are assumed to be constants $t$ and $r$. The angular distribution of each localized inelastic scattering event can be approximately characterized by [14]

$$
L(\theta)=1 /\left[\theta_{x}^{2}+\left(\theta_{z}-\theta_{\mathrm{B}}\right)^{2}+\theta_{\mathrm{E}}^{2}\right]
$$

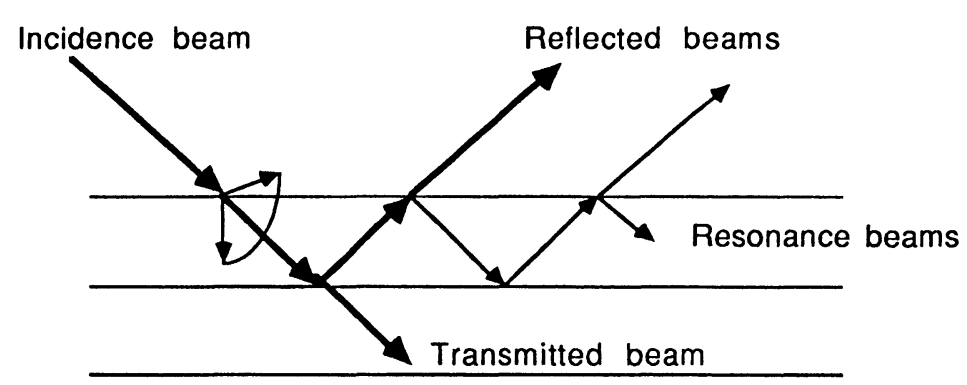

Fig. 8. - A schematic diagram showing the Bragg-resonance reflection processes of high energy electrons from a crystal surface with atomic planes parallel to the surface [13]. Note the electron incidence angle is the Bragg angle if the refraction effect is neglected.

where $\theta_{x}$ and $\theta_{z}$ are the scattering angles parallel and normal to the surface, respectively, and $\theta_{\mathrm{B}}$ is the Bragg angle at which the electrons are incident. Thus the inelastic events occurring in the first layer when the electrons are being Bragg reflected towards the vacuum will give an angular distribution $L(\theta)$ around the specularly reflected spot. Electrons inelastically scattered in the second layer will be (eventually) reflected to vacuum only if they satisfy the Bragg reflection conditions. These electrons will be distributed in a narrow band with approximately the same width as the Kikuchi lines $\left(\Delta \theta_{\mathrm{K}}\right)$ in a form of $L(\theta)$. Electrons penetrating through the second layer into the crystal will be considered as being absorbed. By considering the multiple elastic Bragg scattering and single-incoherent inelastic scattering of the electrons within these two layers and assuming that the inelastic scattering is localized, the final angular distribution of the inelastically scattered electrons within the reflected spot, if $\theta_{\mathrm{E}}>\Delta \theta_{\mathrm{K}}$, may be approximately written as

$$
f(\theta)=\left[\frac{t^{2} r}{1-r^{2}}\right]\left\{\frac{1}{\theta_{x}^{2}+\left(\theta_{z}-\theta_{\mathrm{B}}\right)^{2}+\theta_{\mathrm{E}}^{2}}+\frac{\Delta \theta_{\mathrm{K}} \delta\left(\theta_{z}-\theta_{\mathrm{B}}\right)}{\theta_{x}^{2}+\left(\theta_{z}-\theta_{\mathrm{B}}\right)^{2}+\theta_{\mathrm{E}}^{2}}\right\} .
$$


Integrating $f(\theta)$ for a collection aperture of radius $\beta$ centered on $\theta_{z}=\theta_{\mathrm{B}}$ and $\theta_{x}=0$, one obtains

$$
F(\beta)=\left[\frac{t^{2} r}{1-r^{2}}\right] \ln \left(1+\beta^{2} / \theta_{\mathrm{E}}^{2}\right)+\left[\frac{t^{2} r}{1-r^{2}}\right] \frac{2 \Delta \theta_{\mathrm{K}}}{\theta_{\mathrm{E}}} \arctan \left(\frac{\beta}{\theta_{\mathrm{E}}}\right)
$$

where the first term gives the isolated-atom Lorentzian distribution and the second term gives the deviation caused by diffraction effects. Thus the $K$ factor defined in equation (4) is

$$
K=1+\frac{2 \Delta \theta_{\mathrm{K}}}{\theta_{\mathrm{E}}} \arctan \left(\frac{\beta}{\theta_{\mathrm{E}}}\right) / \ln \left(1+\beta^{2} / \theta_{\mathrm{E}}^{2}\right)
$$

It is impossible, for a general case, to give an analytical expression for $f(\theta)$, because $f(\theta)$ is determined partially by dynamical scattering effects and partially by inelastic multiple plasmon excitations and thermal diffuse scattering [15]. At the present time, it is thus necessary to measure the EICS experimentally.

7.2 A METHOD FOR MEASURING EICS IN TRANSMISSION EXPERIMENTS. - As shown by several authors, the characteristic features in a RHEED pattern can also been observed in a transmission high-energy electron diffraction (THEED) pattern of the same material under identical diffraction conditions $[16,17]$. Thus, it should be possible to define the angular distribution of the inelastically scattered electrons within a reflected spot from measurements made in the transmission geometry on a thin foil of the same crystal with the incidence beam azimuth tilted to set up conditions equivalent to those in the RHEED case. For a specimen with thickness equal to the mean distance $D$ that electrons travel along the surface in the RHEED case, the effects of dynamical scattering and channeling (or resonance) in the RHEED geometry should be equivalently generated in the THEED geometry and thus be automatically included in EELS measurements of EICS ratios for the equivalent diffraction spot. It is expected that the accuracy of REELS microanalysis can be significantly improved using the EICS determined from this method. Further REELS investigations of $\mathrm{MgO}(100)$ with the beam azimuth near [011] will be reported separately [18].

\section{Conclusions.}

It was found experimentally that in reflection electron energy-loss spectroscopy (REELS) for $\mathrm{MgO}(100)$ the optimum signal-to-background ratio at $300 \mathrm{kV}$ was obtained under the following conditions: $\langle 1>\mathbf{g}=(600)$ with beam azimuth a few degrees from [001], in which the electrons are "mirror" reflected under planar resonance conditions; $\langle 2>$ relatively small EELS collection semi-angle $\beta=1.2 \mathrm{mrad}$; and $<3>$ with area selection by the probe rather than the selected area aperture to minimize chromatic aberration effects.

The routine microanalysis formula $I_{\mathrm{A}}(\Delta)=i(\Delta) \sigma(\mathrm{A}, \beta, \Delta) N_{\mathrm{A}} D$ can be applied to REELS surface microanalysis only if $\sigma$ is replaced by the effective ionization cross-section (EICS) $\sigma_{\text {eff }}$, which depends no only on the atomic properties but also on the dynamical electron diffraction and channeling processes from the surface. It should be possible to measure the relative EICS by transmission EELS of a specimen of the same material, with appropriate thickness and under diffracting conditions equivalent to those in the reflection geometry. It is expected that dynamical diffraction and channeling effects should be comprehensively included in this measurement. A $50 \%$ increase in oxygen concentration at a $\mathrm{MgO}(100)$ surface was deduced from REELS experiments under diffraction condition $c$. This result is not affected significantly by the variation of 
diffraction conditions around the [001] zone axis. Channeling effects can produce an incorrect apparent surface composition if different elements are arranged in separated rows when viewed along the beam azimuth.

\section{Acknowledgements.}

This research was sponsored in part by the Division of Materials Sciences, U.S. Department of Energy, under contract DE-AC05-840R21400 with Martin Marietta Energy Systems, Inc. The authors gratefully acknowledge helpful comments of Drs. E.A. Kenik and N.D. Evans, and the practical assistance of Alex Fisher.

\section{References}

[1] WANG Z.L. and COWLEY J.M., Surf. Sci 193 (1988) 501.

[2] WANG Z.L. and BENTLEY J., J. Elect. Microsc. Tech. in press (1990).

[3] WANG Z.L., LIU J., LU P. and COWLEY J.M., Ultramicroscopy 27 (1989) 101.

[4] Bleloch A.L., Howie A. and Lanzerotti M.Y., in Proc. XIIth Intern. Cong. for Electron Microscopy (Seattle) (San Francisco Press) Peachet L.D. and Williams D.B. Eds. 2 (1990) 394.

[5] HowIE A. and MiLNE R.H., J. Microsc. 136 (1984) 279.

[6] EGERTON R.F., Ultramicroscopy 28 (1989) 215.

[7] WANG Z.L., J. Elect. Microsc. Tech. 14 (1990) 13.

[8] NELSON R.L., Disc. Faraday Soc. 41 (1966) 322.

[9] Che M. and TenCH A.J., Advan. Catalysis 32 (1983) 1.

[10] WANG Z.L. and EGERTON R.F., Surf. Sci. 205 (1988) 25.

[11] YaNG Y.Y. and EgerTon R.F., in Proc. XIIth Intern. Cong. for Electron Microscopy (Seattle) (San Francisco Press) Peachey L.D. and Williams D.B. Eds. 3 (1990) 48.

[12] WANG Z.L. and Bentley J., in Proc. XIIth Intern. Cong. for Electron Microscopy (Seattle) (San Francisco Press) Peachey L.D. and Williams D.B. Eds. 2 (1990) 52; WANG Z.L. and BENTLEY J., Ultramicrosc., in press (1990).

[13] WANG Z.L., Micron Microsc. Acta 19 (1988) 201.

[14] see for example EGERTON R.F., Electron energy-loss spectroscopy in the electron microscope (Plenum Press, New York and London, 1986).

[15] WANG Z.L., Surf. Sci. 215 (1989) 201; 217; ibid 214 (1989) 44.

[16 LEHMPFUHL G. and DOWELl W.C.T., Acta Cryst. A42 (1986) 569.

[17] YAO N. and COWLEY J.M., Ultramicroscopy 31 (1989) 149.

[18] WANG Z.L. and BeNTLEY J., in Proc. 49th Annual meeting of EMSA (San Jose) G.W. Bailey Ed. (San Fransisco Press, 1991) in press. 